\title{
Mathematical and computer modeling of intraparticle radionuclides mass transfer in catalytic porous media under isothermal conditions
}

\author{
Vlasyuk A. ${ }^{1}$, Zhukovskyy V. ${ }^{2}$ \\ ${ }^{1}$ The National University of Ostroh Academy \\ 2 Seminarska str., 35800, Ostroh, Ukraine \\ ${ }^{2}$ National University of Water and Environmental Engineering \\ 11 Soborna str., 33000, Rivne, Ukraine
}

(Received 11 November 2017)

\begin{abstract}
The formulation and the mathematical modeling of one-dimensional radionuclides purification process in catalytic porous media under isothermal conditions are considered. The analytical and numerical solutions of the corresponding boundary value problem are found. The comparison of the results is carried out. The "NanoSurface" computer simulation software complex has been improved and the improvement has been verified.
\end{abstract}

Keywords: catalytic porous media, intraparticle mass transfer, verification, NanoSurface.

2000 MSC: $65 \mathrm{~N} 06,35 \mathrm{C} 99,68 \mathrm{~N} 99$

UDC: $532.72: 519.63: 533.15: 631.4$

DOI: $10.23939 / \mathrm{mmc} 2017.02 .117$

\section{Introduction}

The anthropogenic factor of human activity leads to pollution of the biosphere, in particular of fertile lands, with radioactive substances. After the Chornobyl catastrophe, contamination by radionuclides is still observed in the soils of affected areas. Specific natural conditions in the north of the Rivne region contribute to the increasing migration of radionuclides from the soil into plants and by the trophic chains further into the human body. However, modern mechanization accessories can accelerate the purification process of contaminated areas through the use of state-of-the-art melioration technologies, in particular filtering filters, non-traditional organic fertilizers with sorbent microparticles [1], etc.

Scientists consider the motion of colloids in mesoporous and microporous media, where the diameter of pores ranges from 2 to $50 \mathrm{~nm}$ and less than $2 \mathrm{~nm}$, respectively. Colloids (nanoparticles) in soil are soil-forming mineral fragments, mineral precipitates, microorganisms, artificial elements, etc. Their dimensions range from 1 to $1000 \mathrm{~nm}$ [2]. The transport properties of colloids in combination with vertical migration of radionuclides leads to a sharp change in the migration behavior. And it is important to investigate intraparticle mass transfer processes for a better understanding of more complex processes.

Mathematical modeling with the use of adsorbent colloids in the processes of soil purification is a new one. Therefore, in this paper we will consider the mathematical model of only intraparticle mass transfer of radionuclides (contaminant) in the catalytic porous medium, which can be used in more complex statements of underground modeling processes problems [3-5].

The purpose of this work is to find the analytical and numerical solutions of the boundary value problem describing the intraparticle mass transfer of substances in the catalytic porous medium, comparing the results obtained and verifying the created software.

This work was supported by grant of the State Fund for Fundamental Research of Ukraine (grant No $\Phi 76 / 93-2017 / 6$ 04) "Information provision of development of competitive organic agriculture of Ukraine in the conditions of European integration" 


\section{Formulation of the problem}

Let us consider the catalytic porous medium in the domain $\Omega=\{0<x<l ; 0<r<R\}$ (see Fig. 1) with adsorption process of pollutants (radionuclides).

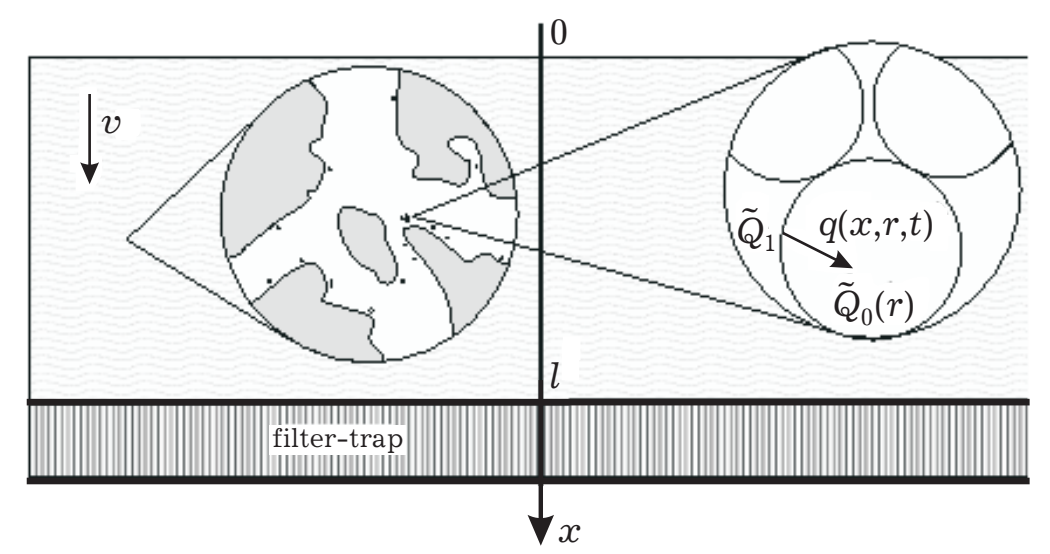

Fig. 1. Vertical radionuclides migration in catalytic porous media.

It is necessary to investigate the distribution of concentration $q(x, r, t)$ in the domain $\Omega$ in time. It will allow us to predict the possibility of using the special micro- and nanoporous particles for soil purification.

\section{Mathematical model}

The mathematical model of the formulated above problem in the one-dimensional case can be described by the following boundary problem $[3,6,7]$ :

$$
\begin{gathered}
\frac{\partial q}{\partial t}=\frac{1}{r^{2}} \frac{\partial}{\partial r}\left(r^{2} D_{0}(q) \frac{\partial q}{\partial r}\right), X \in \Omega, r \in(0, R), t>0 \\
q(x, r, 0)=\tilde{Q}_{0}(r), X \in \Omega, r \in(0, R) \\
q(x, R, t)=0, X \in \Omega, t>0 .
\end{gathered}
$$

where $X=\{x\}$ is the point in the domain $\Omega, r$ is the particle radius, $t$ is the time; $q(x, r, t)$ is the radionuclides concentration in particles with $R$ radius; $D_{0}$ is the diffusion coefficient of radionuclides in particles with radius $R$ located in soil skeleton.

Eq. (1) describes intraparticle radionuclides migration with concentration $q(x, r, t) ;(2)$ - initial concentration $q$ at the start time point; $(3)$ - the initial concentration $q$ on the particle boundary when $r=R$.

In the case when $D_{0}(q)=D_{0}=$ const, Eq. (1) takes the following form

$$
\frac{\partial q}{\partial t}=\frac{1}{r^{2}} \frac{\partial}{\partial r}\left(r^{2} D_{0} \frac{\partial q}{\partial r}\right)
$$

Thus, we can obtain:

$$
\frac{\partial q}{\partial t}=D_{0}\left(\frac{\partial^{2} q}{\partial r^{2}}+\frac{2}{r} \frac{\partial^{2} q}{\partial r}\right), X \in \Omega, r \in(0, R), t>0 .
$$




\section{Analytical solution}

The solution (4) with appropriate boundary conditions (2) and (3) we can find in analytical way [8]:

$$
q(x, r, t)=\frac{1}{r} \sum_{n=1}^{\infty} \beta_{n} e^{-\frac{n^{2} \pi^{2} D_{0} t}{R^{2}}} \sin \frac{n \pi r}{R},
$$

where $\beta_{n}=\frac{2}{R} \int_{0}^{R} r \tilde{Q}_{0}(r) \sin \frac{n \pi r}{R} d r$.

Let us find the analytical solution of Eq. (4) with the boundary condition (2) for the case where the boundary condition (3) is not homogeneous and takes the following form

$$
q(x, R, t)=\tilde{Q}_{1}
$$

where $\tilde{Q}_{1}=$ const.

To reduce the nonhomogeneous boundary condition to homogeneous boundary condition, let us use the following substitution

$$
q(x, r, t)=u(x, r, t)+\tilde{Q}_{1},
$$

where $u(x, r, t)$ is the unknown function.

Then

$$
\begin{gathered}
\frac{\partial q}{\partial t}=\frac{\partial u}{\partial t}, \quad \frac{\partial^{2} q}{\partial r^{2}}=\frac{\partial^{2} u}{\partial r^{2}}, \quad \frac{\partial q}{\partial r}=\frac{\partial u}{\partial r} \\
q(x, R, t)=u(x, R, t)+\tilde{Q}_{1}=\tilde{Q}_{1} \\
q(x, r, 0)=u(x, r, 0)+\tilde{Q}_{1}=\tilde{Q}_{0}(r) .
\end{gathered}
$$

And hence

$$
\begin{gathered}
u(x, R, t)=0, \\
u(x, r, 0)=\tilde{Q}_{0}(r)-\tilde{Q}_{1} .
\end{gathered}
$$

Thus, we have the following boundary value problem for the function $u(x, r, t)$ :

$$
\begin{gathered}
\frac{\partial u}{\partial t}=D_{0}\left(\frac{\partial^{2} u}{\partial r^{2}}+\frac{2}{r} \frac{\partial u}{\partial r}\right), X \in \Omega, r \in(0, R), t>0, \\
u(x, r, 0)=\tilde{Q}_{0}(r)-\tilde{Q}_{1}, X \in \Omega, r \in(0, R), \\
u(x, R, t)=0, X \in \Omega, t>0 .
\end{gathered}
$$

And the solution of (8)-(10) is the following:

$$
u(x, r, t)=\frac{1}{r} \sum_{n=1}^{\infty} \beta_{n} e^{-\frac{n^{2} \pi^{2} D_{0} t}{R^{2}}} \sin \frac{n \pi r}{R},
$$

where $\beta_{n}=\frac{2}{R} \int_{0}^{R} r\left(\tilde{Q}_{0}(r)-\tilde{Q}_{1}\right) \sin \frac{n \pi r}{R} d r$.

Returning to the replacement (7), we obtain the analytical solution (4) with the appropriate boundary conditions (2) and (6) in the form of the following function:

$$
q(x, r, t)=\tilde{Q}_{1}+\frac{1}{r} \sum_{n=1}^{\infty} \beta_{n} e^{-\frac{n^{2} \pi^{2} D_{0} t}{R^{2}}} \sin \frac{n \pi r}{R},
$$

where $\beta_{n}=\frac{2}{R} \int_{0}^{R} r\left(\tilde{Q}_{0}(r)-\tilde{Q}_{1}\right) \sin \frac{n \pi r}{R} d r$. 


\section{Numerical solution of the boundary value problem}

For the numerical solving the boundary value problem (1)-(3), we use the finite-difference method with the monotone difference scheme [9].

Let us cover the area $\Omega$ with a uniform grid $\omega_{h_{1} h_{2} \tau}$ with the steps $h_{1}, h_{2}$ and $\tau$ along the axes $O x$, Or respectively, and the time $O t$ for the variables $x, r, t$

$$
\omega_{h_{11} h_{12} h_{2} \tau}=\left\{\begin{array}{c}
x_{i_{1}}=i_{1} h_{1}, r_{j}=j h_{2}, t_{k}=k \tau, \\
i_{1}=\overline{0, n_{1}}, j=\overline{0, n_{2}}, k=\overline{0, n_{3}}, \\
h_{1} n_{1}=l_{1}, h_{2} n_{2}=R, \tau n_{3}=T,
\end{array}\right\},
$$

where $n_{1}, n_{2}, n_{3}$ are the numbers of steps for spatial variables and time respectively.

Finite-difference analog of the differential equation (4) has the following form:

$$
\begin{aligned}
\frac{q_{j}^{(k+1)}-q_{j}^{(k)}}{\tau^{\prime}} & =\tilde{\chi} \frac{q_{j+1}^{(k+1)}-2 q_{j}^{(k+1)}+q_{j-1}^{(k+1)}}{h_{2}^{2}}+\frac{\tilde{r}_{+}}{D_{0}} \frac{q_{j+1}^{(k+1)}-q_{j}^{(k+1)}}{h_{2}}+\frac{\tilde{r}_{-}}{D_{0}} \frac{q_{j}^{(k+1)}-q_{j-1}^{(k+1)}}{h_{2}}, \\
j & =\overline{1, n_{2}-1}, \quad k=\overline{0, n_{3}},
\end{aligned}
$$

where

$$
\begin{aligned}
& \tilde{\chi}=\frac{1}{1+\frac{h_{2}|\tilde{r}|}{2 D_{0}}}=1-\frac{h_{2}|\tilde{r}|}{2 D_{0}}+O\left(h_{2}^{2}\right), \quad \tilde{r}=\frac{2}{r}, \quad \tau^{\prime}=D_{0} \tau, \\
& \tilde{r}_{+}=\frac{-\tilde{r}+|\tilde{r}|}{2} \geqslant 0, \quad \tilde{r}_{-}=\frac{-\tilde{r}-|\tilde{r}|}{2} \geqslant 0, \quad \tilde{r}=\tilde{r}_{+}+\tilde{r}_{-} .
\end{aligned}
$$

For the initial condition (2) we obtain the following finite-difference analog:

$$
q_{j}^{(0)}=\tilde{Q}_{0}\left(j h_{2}\right), \quad j=\overline{1, n_{2}-1}
$$

For the homogeneous first-type boundary condition (3) we have

$$
q_{n_{2}}^{(k)}=0, \quad k=\overline{1, n_{3}}
$$

but for the nonhomogeneous first-type boundary condition (6)

$$
q_{n_{2}}^{(k)}=\tilde{Q}_{1}, \quad k=\overline{1, n_{3}},
$$

This system (13) can be solved using the sweep method in the form of recurrence relations

$$
a_{j}^{1} q_{j-1}^{(k+1)}-\bar{c}_{j}^{1} q_{j}^{(k+1)}+b_{j}^{1} q_{j+1}^{(k+1)}=-q_{j}^{(k)},
$$

where

$$
a_{j}^{1}=\tau^{\prime}\left(\frac{\bar{\chi}}{h_{2}^{2}}-\frac{\tilde{r}_{-}}{h_{2} D_{0}}\right), \quad b_{j}^{1}=\tau^{\prime}\left(\frac{\bar{\chi}}{h_{2}^{2}}+\frac{\tilde{r}_{+}}{h_{2} D_{0}}\right), \quad \bar{c}_{j}^{1}=1+\tau^{\prime}\left(\frac{2 \bar{\chi}}{h_{2}^{2}}+\frac{1}{h_{2} D_{0}}\left(\tilde{r}_{+}-\tilde{r}_{-}\right)\right) .
$$

It can be shown that the stability conditions for the finite-difference scheme $\left|\bar{c}_{j}^{1}\right|>\left|a_{j}^{1}\right|+\left|b_{j}^{1}\right|$ are satisfied. The concentration value $q(x, r, t)$ at the time level $(k+1)$ we can find by the sweep method

$$
q_{j}^{(k+1)}=\alpha_{j+1}^{1} q_{j+1}^{(k+1)}+\beta_{j+1}^{1},
$$

where $\alpha_{j+1}^{1}=\frac{b_{j}^{1}}{\bar{c}_{j}^{1}-\alpha_{j}^{1} a_{j}^{1}}, \beta_{j+1}^{1}=\frac{a_{j}^{1} \beta_{j}^{1}+q_{j}^{(k)}}{\bar{c}_{j}^{1}-\alpha_{j}^{1} a_{j}^{1}}, j=\overline{1, n_{2}-1}, k=\overline{1, n_{3}}$. 
For the discretization equation (1) let us use the implicit difference scheme

$$
\frac{q_{j}^{(k+1)}-q_{j}^{(k)}}{\tau}=\frac{1}{r^{2} h_{2}}\left(\left(d_{0}\right)_{j+1}^{(k)} \frac{q_{j+1}^{(k+1)}-q_{j}^{(k+1)}}{h_{2}}-\left(d_{0}\right)_{j}^{(k)} \frac{q_{j}^{(k+1)}-q_{j-1}^{(k+1)}}{h_{2}}\right)
$$

where $\left(d_{0}\right)_{j}^{(k)}=\frac{r^{2}}{2}\left(\left(D_{0}\right)_{j}^{(k)}+\left(D_{0}\right)_{j-1}^{(k)}\right), j=\overline{1, n_{2}-1}, k=\overline{0, n_{3}}$.

The concentration value $q(x, r, t)$ we find by solving the finite-difference equation (18) with the appropriate boundary conditions. To do this, let us reduce this equation to the sweep method form

$$
a_{j}^{2} q_{j-1}^{(k+1)}-\bar{c}_{j}^{2} q_{j}^{(k+1)}+b_{j}^{2} q_{j+1}^{(k+1)}=-q_{j}^{(k)},
$$

where

$$
a_{j}^{2}=\frac{\tau}{r^{2} h_{2}^{2}}\left(d_{0}\right)_{j}^{(k)}, \quad b_{j}^{2}=\frac{\tau}{r^{2} h_{2}^{2}}\left(d_{0}\right)_{j+1}^{(k)}, \quad \bar{c}_{j}^{2}=1+\frac{\tau}{r^{2} h_{2}^{2}}\left(\left(d_{0}\right)_{j}^{(k)}+\left(d_{0}\right)_{j+1}^{(k)}\right) .
$$

It can be shown that the stability conditions for the finite-difference scheme $\left|\bar{c}_{j}^{2}\right|>\left|a_{j}^{2}\right|+\left|b_{j}^{2}\right|$ are satisfied. The concentration value $q(x, r, t)$ at the time level $(k+1)$ is sequentially calculated using the ratio

$$
q_{j}^{(k+1)}=\alpha_{j+1}^{2} q_{j+1}^{(k+1)}+\beta_{j+1}^{2},
$$

where $\alpha_{j+1}^{2}=\frac{b_{j}^{2}}{\bar{c}_{j}^{2}-\alpha_{j}^{2} a_{j}^{2}}, \beta_{j+1}^{2}=\frac{a_{j}^{2} \beta_{j}^{2}+q_{j}^{(k)}}{\bar{c}_{j}^{2}-\alpha_{j}^{2} a_{j}^{2}}, j=\overline{1, n_{2}-1}, k=\overline{1, n_{3}}$.

For the discretization equation (1) with monotone finite difference scheme let us do the following math transformation. From Eq. (1) it follows that

$$
\frac{\partial q}{\partial t}=\frac{1}{r^{2}} r^{2} D_{0}(q) \frac{\partial^{2} q}{\partial r^{2}}+\frac{1}{r^{2}} \frac{\partial}{\partial r}\left(r^{2} D_{0}(q)\right) \frac{\partial q}{\partial r}
$$

or

$$
\frac{\partial q}{\partial t}=D_{0}(q) \frac{\partial^{2} q}{\partial r^{2}}+\frac{1}{r^{2}}\left(2 r D_{0}(q)+r^{2} \frac{\partial D_{0}(q)}{\partial r}\right) \frac{\partial q}{\partial r}
$$

Then

$$
\frac{\partial q}{\partial t}=D_{0}(q) \frac{\partial^{2} q}{\partial r^{2}}+\left(\frac{D_{0}(q)}{r}+\frac{\partial D_{0}(q)}{\partial r}\right) \frac{\partial q}{\partial r}
$$

And hence

$$
\frac{\partial q}{\partial t}=D_{0}(q) \frac{\partial^{2} q}{\partial r^{2}}+p(q) \frac{\partial q}{\partial r}
$$

where $p(q)=\frac{D_{0}(q)}{r}+\frac{\partial D_{0}(q)}{\partial r}$.

The finite-difference analog of the differential equation (20) has the form

$$
\begin{gathered}
\frac{q_{j}^{(k+1)}-q_{j}^{(k)}}{\tau^{\prime \prime}}=\hat{\chi}_{j}^{(k)} \frac{q_{j+1}^{(k+1)}-2 q_{j}^{(k+1)}+q_{j-1}^{(k+1)}}{h_{2}^{2}}+\left(\hat{r}_{+}\right)_{j}^{(k)} \frac{q_{j+1}^{(k+1)}-q_{j}^{(k+1)}}{h_{2}}+\left(\hat{r}_{-}\right)_{j}^{(k)} \frac{q_{j}^{(k+1)}-q_{j-1}^{(k+1)}}{h_{2}} \\
j=\overline{1, n_{2}-1}, \quad k=\overline{0, n_{3}},
\end{gathered}
$$

where

$$
\begin{gathered}
\hat{\chi}_{j}^{(k)}=\frac{1}{1+0,5 h_{1}\left|\hat{r}_{j}^{(k)}\right|}, \quad \hat{r}_{j}^{(k)}=\frac{p_{j}^{(k)}}{\left(D_{0}\right)_{j}^{(k)}}, \quad \tau^{\prime \prime}=\tau\left(D_{0}\right)_{j}^{(k)},\left(\hat{r}_{+}\right)_{j}^{(k)}=\frac{-\hat{r}_{j}^{(k)}+\left|\hat{r}_{j}^{(k)}\right|}{2} \geqslant 0, \\
\left(\hat{r}_{-}\right)_{j}^{(k)}=\frac{-\hat{r}_{j}^{(k)}-\left|\hat{r}_{j}^{(k)}\right|}{2} \leqslant 0, \quad \hat{r}_{j}^{(k)}=\left(\hat{r}_{+}\right)_{j}^{(k)}+\left(\hat{r}_{-}\right)_{j}^{(k)} .
\end{gathered}
$$


With the sweep method, Eq. (21) can be rewritten in the form

$$
a_{j}^{3} q_{j-1}^{(k+1)}-\bar{c}_{j}^{3} q_{j}^{(k+1)}+b_{j}^{3} q_{j+1}^{(k+1)}=-q_{j}^{(k)},
$$

in this equation

$$
a_{j}^{3}=\frac{\hat{\chi}_{j}^{(k)}}{h_{2}^{2}}-\frac{\left(\hat{r}_{-}\right)_{j}^{(k)}}{h_{2}}, \quad b_{j}^{3}=\frac{\hat{\chi}_{j}^{(k)}}{h_{2}^{2}}+\frac{\left(\hat{r}_{+}\right)_{j}^{(k)}}{h_{2}}, \quad \bar{c}_{j}^{3}=\frac{2 \hat{\chi}_{j}^{(k)}}{h_{2}^{2}}+\frac{\left(\hat{r}_{+}\right)_{j}^{(k)}}{h_{2}}-\frac{\left(\hat{r}_{-}\right)_{j}^{(k)}}{h_{2}} .
$$

It can be shown that the stability conditions for the finite-difference scheme $\left|\bar{c}_{j}^{3}\right|>\left|a_{j}^{3}\right|+\left|b_{j}^{3}\right|$ are satisfied. The concentration value $q(x, r, t)$ at the time level $(k+1)$ we consistently calculate using the ratio

$$
q_{j}^{(k+1)}=\alpha_{j+1}^{3} q_{j+1}^{(k+1)}+\beta_{j+1}^{3},
$$

where $\alpha_{j+1}^{3}=\frac{b_{j}^{3}}{\bar{c}_{j}^{3}-\alpha_{j}^{3} a_{j}^{3}}, \beta_{j+1}^{3}=\frac{a_{j}^{3} \beta_{j}^{3}+q_{j}^{(k)}}{\bar{c}_{j}^{3}-\alpha_{j}^{3} a_{j}^{3}}, j=\overline{1, n_{2}-1}, k=\overline{1, n_{3}}$.

\section{Software implementation}

The program implementation of the above-mentioned analytical and numerical solutions of the problem of adsorption mass transfer in the catalytic porous medium is presented in the form of new mathematical models of "NanoSurface" program complex (see Fig. 2). This cross-platform product is written in the $\mathrm{C} / \mathrm{C}++$ programming language with the use of the Qt framework and designed for computer simulation of the problems of mass transfer of saline solutions in catalytic and dispersed media of micro- and nanoporous structure particles using parallel computations [10].

\section{Experiments and their analysis}

The results of computer modeling of intraparticular mass transfer of radionuclides in the catalytic porous medium are obtained using both the analytic solutions of the problem (1)-(3) and using the numerical solution for the concentration values.

Analytical methods of investigation of mass transfer equations have been developing for a long time, but despite this there is a limited number of tasks that can be solved analytically. The range of potential problems has considerably expanded with the development of computer technology and numerical methods that allow us to obtain a solution with a given accuracy. An analytical solution is usually found for a simplified version of the task (mathematical model), which allows one to understand the physical essence of the process, as well as helps to test the numerical algorithm and verify the program code.

Verification of the code includes the verification of compliance with the basic rules for the design of software systems: system architecture, programming languages, development methodology, version control systems, quality assurance management [11]. Most of these requirements refer to the standard software development issues [12]. Another part of the verification rules is related to the scientific calculations, where the main task is to verify the accuracy of mathematical model representation in the form of the code [13]. Mathematical models are systems of differential equations with corresponding boundary conditions, where the sweep method with ensured stability conditions is used to find a numerical solution [3,14]. That is, the correctness of the computational algorithm is ensured by a clear statement of the boundary value problem and the choice of the method of computation. However, concerning this, we cannot be sure that the obtained results do not contain errors or the program logical "bugs".

Numerical experiments were carried out with the following geometric and adsorption parameters: $R=5 \cdot 10^{-8} \mathrm{~m}, D_{0}=1 \cdot 10^{-18} \frac{\mathrm{m}^{2}}{\text { day }}$. 


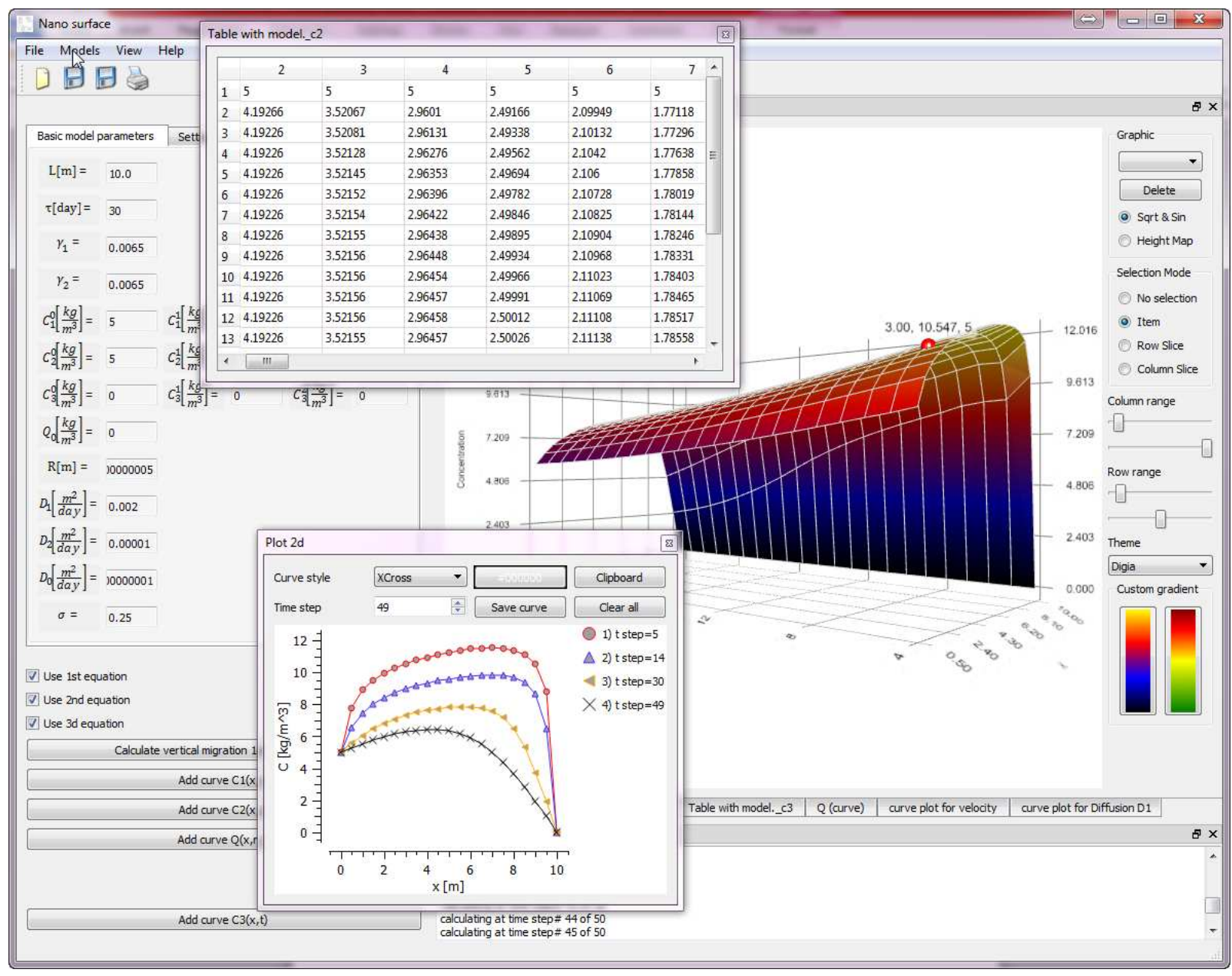

Fig. 2. Interface of "Nanosurface" software complex.

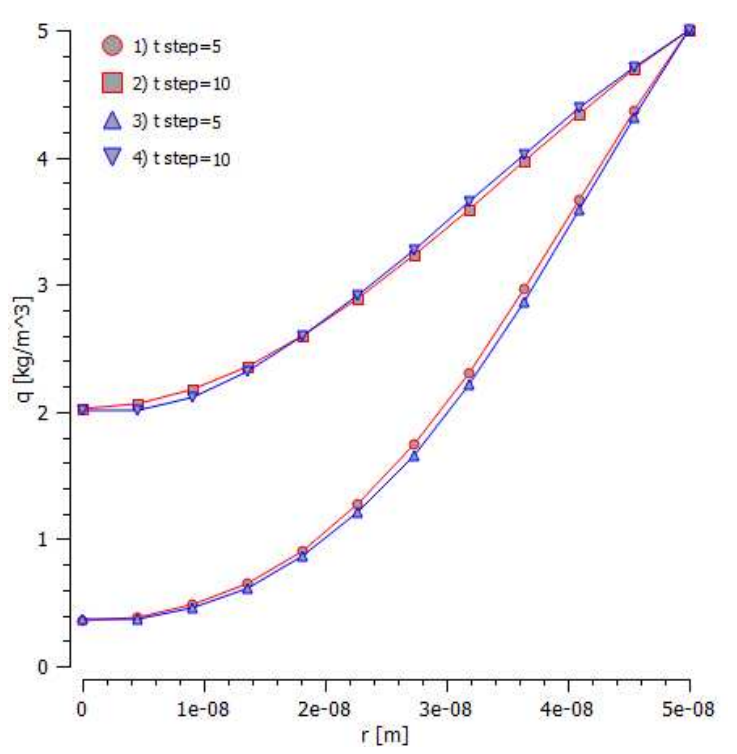

$a$

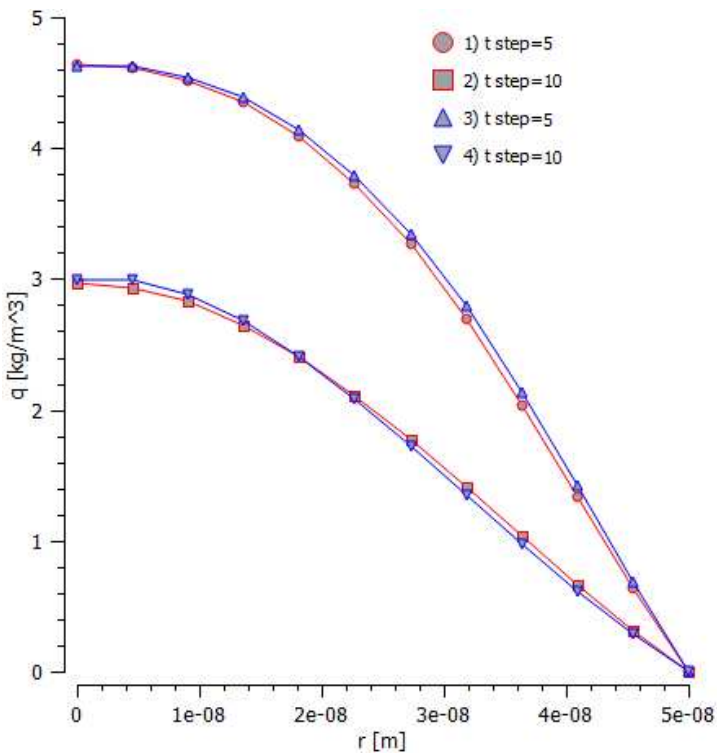

$b$

Fig. 3. Comparative distribution of concentration $q(r)$ for different particle radius $r$ for analytical (curves 1 and 2) and numerical (curves 3 and 4) solutions at different time steps: 1) and 3 ) $t=5$ months, 2) and 4 ) $t=10$ months and different boundary conditions: a) $\left.\tilde{Q}_{1}=5 \frac{\mathrm{kg}}{\mathrm{m}^{3}}, \tilde{Q}_{0}(r)=0 \frac{\mathrm{kg}}{\mathrm{m}^{3}} ; \mathrm{b}\right) \tilde{Q}_{1}=0 \frac{\mathrm{kg}}{\mathrm{m}^{3}}, \tilde{Q}_{0}(r)=5 \frac{\mathrm{kg}}{\mathrm{m}^{3}}$. 
Fig. 3 shows a comparative analysis of the results obtained by the analytical and the numerical methods under different initial and boundary conditions. As can be seen from the figure, the difference in the results does not exceed $0.5 \%$. From this, we can conclude that the problem statement is correct and the software solution is successfully verified.

\section{Conclusions}

We have considered a mathematical model for intraparticle migration of radionuclides in catalytic porous media. It was obtained the numerical solution of the corresponding one-dimensional boundary value problem by the method of finite differences as well as the analytical solution. We used implicit and monotone difference schemas for diffusion-convection problems. At the same time we proved numerical stability. Using created "NanoSurface" software a series of numerical experiments and their analysis were conducted. Comparison of the the results obtained by analytical and numerical methods allows us to successfully verify created software.

[1] Auffan M., Shipley H. J., Yean S., Kan A. T., Tomson M., Rose J., Bottero J. Y. Nanomaterials as adsorbents, Environmental Nanotechnology: Applications and Impacts of Nanomaterials. McGraw-Hill, New York, pp. 371-392 (2007).

[2] Rouquerol J., Avnir D., Fairbridge C.W., EverettD. H., Haynes J. M., PerniconeN., Ramsay J.D.F., Sing K. S. W., Unger K. K. Recommendations for the characterization of porous solids (Technical Report). Pure Appl. Chem. 66 (8), 1739-1758 (1994).

[3] Vlasyuk A. P., Zhukovskyy V. V., Bondarchuk M. M. Mathematical Modelling of Vertical Migration of Radionuclides in Catalytic Porous Media with Traps in Linear Case, Theoretical and Applied Aspects of Cybernetics. Proceedings of the 5th International Scientific Conference of Students and Young Scientists, pp. 208-219 (2015).

[4] Safonyk A.P. Modelling the filtration processes of liquids from multicomponent contamination in the conditions of authentication of mass transfer coefficient. International Journal of Mathematical Models and Methods in Applied Sciences. 9, 189-192 (2015).

[5] Vlasyuk A. P., Zhukovskii V.V. Mathematical Simulation of the Migration of Radionuclides in a Soil Medium Under Nonisothermal Conditions with Account for Catalytic Microparticles and Nonlinear Processes. Journal of Engineering Physics and Thermophysics. 90 (6), 1386-1398 (2017).

[6] Conner W. C., Fraissard J. P. Fluid transport in nanoporous materials, Vol. 219 of NATO science series II: Mathematics, physics, and chemistry, Springer in cooperation with NATO Public Diplomacy Division, Dordrecht, the Netherlands (2006).

[7] Petryk M., Leclerc S., Canet D., Sergienko I., Deineka V., Fraissard J. Competitive Diffusion of Gases in a Zeolite Bed: NMR and Slice Selection Procedure, Modeling, and Parameter Identification. The Journal of Physical Chemistry C. 119 (47), 26519-26525 (2015).

[8] Budak B. M., Samarskii A. A., Tikhonov A. N., Sneddon I. N., Stark M., Ulam S. A Collection of Problems on Mathematical Physics: International Series of Monographs in Pure and Applied Mathematics, Elsevier Science (2013).

[9] Marchuk G. I. Methods of Numerical Mathematics, Stochastic Modelling and Applied Probability. Springer Verlag, New York, NY (1982).

[10] Vlasyuk A. P., Zhukovskyy V. V. Nanosurface - a tool for computer modeling of mass transfer process in catalytic porous media. Abstracts of XXVIII International Conference "Problems of decision making under uncertainties", pp. 122-124 (2016).

[11] Vlasyuk A. P., Zhukovskyy V.V. A modern approach for software construction of tools for mathematical modeling of mass transfer processes in catalytic porous media. Theoretical \& Applied Science. 44 (12), 69-75 (2016).

[12] McConnell S. Code complete. Microsoft Press, Redmond, Wash., 2nd edition edition (2004).

[13] Oberkampf W. L. Verification and validation in scientific computing. Cambridge Univ. Press, Cambridge [u.a.] (2013). 
[14] Zhukovskyy V.V., Vlasyuk A.P. Mathematical modelling of vertical migration of radionuclides in catalytic porous media in non-isothermal conditions. Research on modern systems for manufacture and measurement of components of machines and devices, SCIENCE REPORT Project CIII-PL-0007, 177-190 (2016).

\title{
Математичне і комп'ютерне моделювання внутрішньочастинкового масоперенесення радіонуклідів у каталітичному пористому середовищі за ізотермічних умов
}

\author{
Власюк А. ${ }^{1}$, Жуковський В. ${ }^{2}$ \\ ${ }^{1}$ Національний університет «Острозъка академія» \\ вул. Семінарсъка, 2, 35800, Острог, Україна \\ ${ }^{2}$ Національний університет водного господарства та природокористування \\ вул. Соборна, 11, 35000, Рівне, Україна
}

Розглянуто постановку та проведено математичне моделювання одновимірної задачі адсорбційного масоперенесення радіонуклідів у каталітичному пористому середовищі за ізотермічних умов. Знайдено аналітичний та чисельний розв'язок відповідної крайової задачі. Порівняно отримані результати. Удосконалено програмний комплекс комп'ютерного моделювання NanoSurface та проведено його верифікацію.

Ключові слова: каталітичне пористе середовище, внутрішнъочастинкове масоперенесення, верифікація, NanoSurface.

2000 MSC: $65 \mathrm{~N} 06,35 \mathrm{C} 99,68 \mathrm{~N} 99$

УдК: 532.72:519.63:533.15:631.4 